\title{
EIN NEUES AUTOGRAPHON DES NIKOLAOS KABASILAS: DER KODEX VATIC. PALAT. GR. 211*
}

\author{
Mit zwei Tafeln
}

Im Jahre 1960, drei Jahre nach seiner Ausgabe des 'Anti-Zealot'-Traktats des Nikolaos Kabasilas ${ }^{1}$, präsentierte I. Ševčenko die ff. $65-87$ und 96-99 des Kodex Paris. gr. 1276 als ein Arbeitsexemplar des Autors für diesen Traktat ${ }^{2}$. Ševčenko behauptete mit Recht und bewies vorzüglich, daß die Textänderungen und die Ergänzungen, die in margine des Paris. 1276 stehen, nicht von einem Kopisten, sondern nur von der Hand des Autors stammen können ${ }^{3}$.

Die Untersuchung des Kodex Vatic. Palat. gr. 211 bietet m.E. ein ähnliches Beispiel für ein anderes Werk desselben Autors, seine Lobrede auf die heilige Theodora von Thessalonike. Der Kodex, ein kleinformatiges Manuskript aus dem 14. Jh., überliefert einerseits zwei Enkomia des Nikolaos Kabasilas, eines auf den Schutzpatron seiner Heimatstadt, den heiligen Demetrios, und eines auf die ebenfalls in Thessalonike gefeierte heilige Theodora, und andererseits zwei weitere hagiographische Texte ebenfalls

* Den Anlaß für die Beschäftigung mit dem Enkomion auf die hl. Theodora gab mir eine Seminararbeit von Frau Iliana Paraskeuopoulou, der ich dafür herzlich danke.

1 I. ŠEvČenko, Nicolas Cabasilas' "Anti-Zealot" Discourse: A Reinterpretation. DOP 11 (1957) 81-171 (= I. ŠEvČENKo, Society and Intellectual Life in Late Byzantium. London 1981 [Variorum Reprints], IV).

2 I. Šmerčnko, The Author's Draft of Nicolas Cabasilas' "Anti-Zealot" Discourse in Parisinus Graecus 12\%6. DOP 14 (1960) 181-201 (= I. ŠEvČENko, Society and Intellectual Life in Late Byzantium. London 1981 [Variorum Reprints], V).

3 Vgl. ŠEvČEnko, 186: «Hand $\mathrm{A}^{2}$, whose many corrections are largely responsible for the Second Version of the Discourse, is neither that of a scribe nor of a corrector, but rather that of a fourteenth-century editor. An editor whose style and thoughts are the same as those of the text he is editing can only be the author himself. Changes made by hand $\mathrm{A}^{2}$ in the First Version of the Discourse are specimens of the author's own handwriting». Die Identifizierung der Hand $\mathrm{A}^{2}$ mit der des Nikolaos Kabasilas bespricht Ševěenko nochmals in: I. ŠevČenko, A Postscript on Nicolas Cabasilas' "Anti-Zealot" Discourse. DOP 16 (1962) 403-408 (= I. Ševčenko, Society and Intellectual Life in Late Byzantium. London 1981 [Variorum Reprints], VI), hier besonders 406-407. 
für Theodora, nämlich die überarbeitete Fassung ihrer Vita und der Translatio ihrer Reliquien, verfaßt von Gregorios Klerikos ${ }^{4}$.

Der spezielle Inhalt des Kodex sowie eine spätere Notiz aus dem 15. Jh., die von seiner Herkunft aus dem Kloster der heiligen Theodora spricht ${ }^{5}$, weist auf eine Beziehung des Kodex zu der Stadt Thessalonike hin. Der Kodex ist von einer einzigen Hand geschrieben, die sich um die Mitte des 14. Jahrhunderts datieren läßt $\mathrm{t}^{6}$.

Auffällig sind im letzten Teil des Kodex die Spuren einer starken Bearbeitung des tradierten Textes, nämlich der Lobrede des Nikolaos Kabasilas auf die heilige Theodora (BHG 1741). Mehrere Wörter bzw. Zeilen des Textes sind ausradiert und durch einen neuen Text ersetzt, Textpartien sind durch- oder quergestrichen und zahlreiche Ergänzungen sind in margine oder inter lineas geschrieben. Obwohl zwischen dem Schriftstil des Haupttextes und dem der Korrekturen bzw. Ergänzungen ein Unterschied festzustellen ist - der Haupttext zeigt beständig einen ruhigen Schriftduktus, während die Ergänzungen einen kursiveren aufweisen -, besteht kein Zweifel, daß alle diese Änderungen und Ergänzungen von der Hand des Schreibers stammen. Und da sie, genau wie im Fall des Paris. 1276, nicht von einem Kopisten oder einer dritten Person vorgenommen werden konnten, liegt auf der Hand, daß der Schreiber und Korrektor dieses Enkomions und des gesamten Kodex Nikolaos Kabasilas selbst ist. Der kursivere Stil der in margine stehenden Änderungen bzw. Ergänzungen steht dem Stil der Hand $\mathrm{A}^{2}$ (Nikolaos Kabasilas) des Paris. 1276 näher, während der kalligraphische jenem des Paris. 1276, f. $100^{7}$, des Kodex Panagias Kamariotisses (Chalke) $157^{8}$, ff. 294-300v, und möglicherweise des Meteor. Barlaam 202 ähnelt ${ }^{9}$.

${ }^{4}$ Für eine Beschreibung des Kodex s. H. Stevenson, Codices Manuscripti Palatini Graeci Bibliothecae Vaticanae. Romae 1885, 108-109 und Hagiographi Bollandiani-P. Franchi de' Cavalieri, Catalogus codicum hagiographicorum graecorum bibliothecae Vaticanae [Subsidia Hagiographica 7]. Bruxelles 1899, 217.

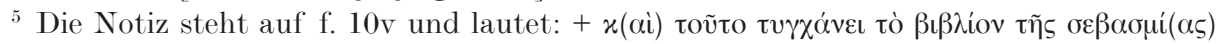

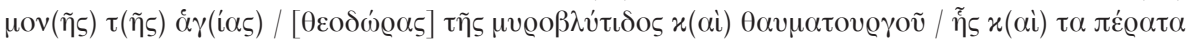

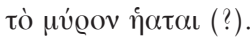

${ }^{6}$ Die Datierung wird auch von den Wasserzeichen bestätigt, die aus derselben Zeit stammen; vgl. unten Anm. 10.

${ }^{7} \mathrm{Zu}$ Ševčenkos Bedenken, die Hand A (d.h. die Hand des Haupttextes) des Paris. 1276 mit der des Nikolaos Kabasilas zu identifizieren, s. ŠEvčEnKo, 201.

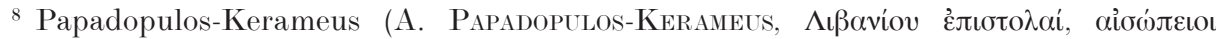

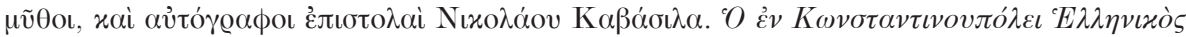

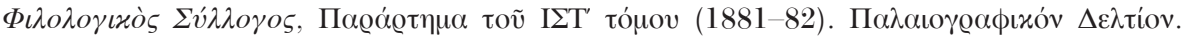
Konstantinopel 1885, 47) hat als erster aufgrund einer Notiz, die von einer anderen Hand stammt und am Beginn der Briefsammlung des Nikolaos Kabasilas steht (f. 294: 
Die Identifizierung des Kopisten mit Nikolaos Kabasilas bestärken weiterhin folgende äußerliche Gegebenheiten: (a) Das Format des Kodex sowie die Mischung von Pergament- und Papierlagen deuten darauf hin, daß der Kodex zum privaten Gebrauch bestimmt war ${ }^{10}$; (b) der Kodex gehörte dem Kloster der heiligen Theodora, in dem die Mutter von Nikolaos Kabasilas als Nonne gelebt hat ${ }^{11}$; (c) beide Enkomia des Nikolaos Kabasilas, die im Kodex enthalten sind, erwähnen nicht in ihrem Titel den Namen ihres Autors, was im Fall eines Autographons überflüssig ist ${ }^{12}$.

Der Vatic. Palat. 211 ist mit Sicherheit der älteste erhaltene Textzeuge für das Enkomion auf die heilige Theodora, nicht nur wegen seiner Datie-

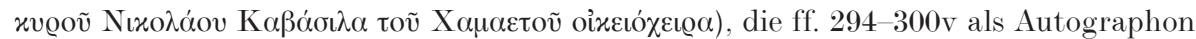
des Kabasilas bezeichnet. Enepekides hat in seiner Ausgabe (s. P. EnepeKides, Der Briefwechsel des Mystikers Nikolaos Kabasilas. Kommentierte Textausgabe. BZ 47 (1953) 18-46, hier besonders 21) mangels eines bekannten Autographon gezögert, sich für oder gegen eine endgültige Zuweisung der Hand zu entscheiden, während ŠEvČENKo (186-187), der zwei Hände (A: f. 294r-294v Z. 1 und B: ff. 295 Z. 2-300v) unterscheidet, Ähnlichkeiten zwischen der Hand $\mathrm{A}^{2}$ des Paris. 1276 (Kabasilas) und Hand B des Kodex Panagias Kamariotisses 157 erkennt. Den Kodex durfte ich mit der Erlaubnis des Ökumenischen Patriarchen von Konstantinopel kurz einsehen, wofür ich ihm meine Dankbarkeit aussprechen möchte; die Kopistenänderung, von der Ševčenko spricht, kann ich leider nicht bestätigen.

9 Der Kodex enthält einen großen Teil des Werkes von Nikolaos Kabasilas und könnte, nach Laurent (s. V. Laurent, Un nouveau témoin de la Correspondance de Démetrius Cydonès et de l'activité littéraire de Nicolas Cabasilas Chamaétos: Le codex Meteor. Barlaam 202. Hell 9 [1936] 188), der den Kodex intensiv studiert hat, als sein Autographon betrachtet werden. Die Zuweisung basiert auf der Ähnlichkeit der Schrift mit der des Chalke-Kodex. Wie schon Ševčenko bemerkt hat, erlaubt der vorsichtige Duktus des Meteor. Barlaam 202 in Vergleich zu dem kursiven des Korrektors (Hand $\mathrm{A}^{2}$ ) keine sichere Aussage. Dr. K. Lappas hat mir freundlicherweise Kopien des Enkomion geschickt und damit meine Untersuchungen wesentlich unterstützt; ihm sei auch an dieser Stelle herzlich gedankt. Zu der textgeschichtlichen Verwandtschaft des Kodex mit dem Vatic. Palat. 211 s. unten.

${ }^{10}$ Das Format ist relativ klein $(202 \times 138 \mathrm{~mm})$ und von den 97 Blättern des Kodex bestehen die ff. 7-92 aus Pergament, während die ff. 1-6 und 93-97, d. h. die erste und letzte Lage aus westlichem Papier mit Wasserzeichen bestehen (Kreise ähnlich wie C. M. Briquet, Les filigranes. Dictionnaire historique des marques du papier dès leur apparition vers 1282 jusqu'en 1600. Genève 1907; Leipzig ${ }^{2} 1923$; The New Briquet, Jubilee Edition. Amsterdam 1968, Nr. 3189, aus dem Jahre 1347).

11 S. oben Anm. 5. Über die Mutter des Nikolaos Kabasilas, die sich eine Zeitlang als Nonne in diesem Kloster aufgehalten hat, informiert uns Georgios Sprantzes; s. Giorgio Sfranze, Cronaca, ed. R. Maisano [CFHB XXIX], Roma 1990, 48.1-3 (= XVIII 2.1-3).

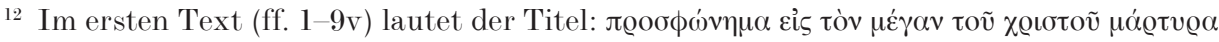

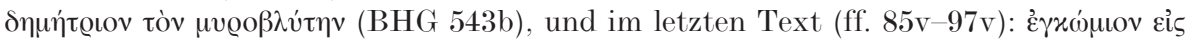

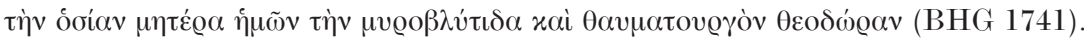


rung in das 14. Jh., sondern auch wegen der Textfassung, die er ante correctionem überliefert (Redaktion $\mathrm{A})^{13}$. Vergleicht man den ursprünglichen Text des Palatinus (Redaktion A) mit dem der anderen Handschriften, ergibt sich eindeutig, daß dieser eine erste Fassung des Textes darstellt, die wesentlich von der von ihnen überlieferten (Redaktion C) abweicht ${ }^{14}$. Letztere stimmt von einigen Ausnahmen abgesehen mit der post correctionem Textfassung des Palatinus (Redaktion B) überein.

Folgende Textstellen, die von der Redaktion C bekannt sind ${ }^{15}$, sind im Palatinus (V) nach Ausradieren des vorherstehenden Textes (Redaktion A) geschrieben:

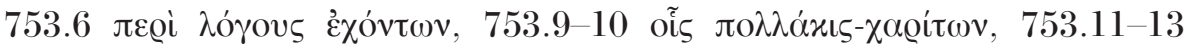

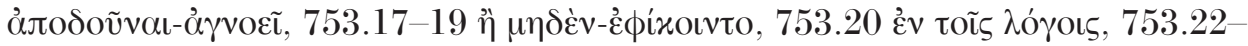

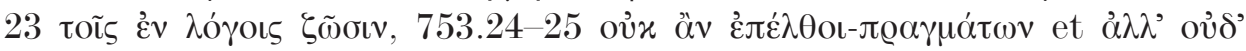

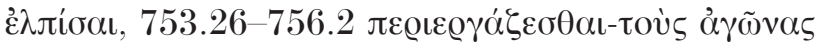

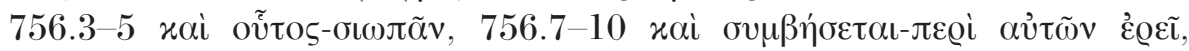

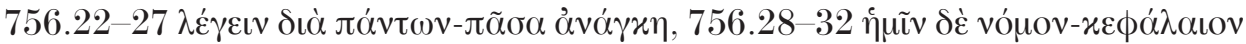

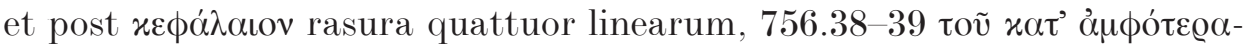

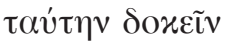

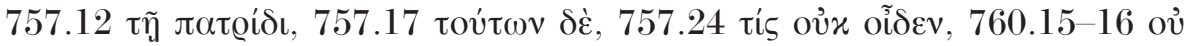

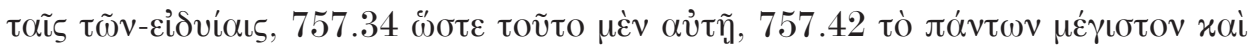

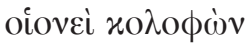

${ }^{13}$ Von den fünf weiteren Handschriften des Enkomions, die bekannt sind (vgl. S. A. PA-

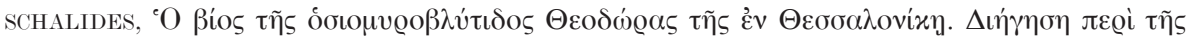

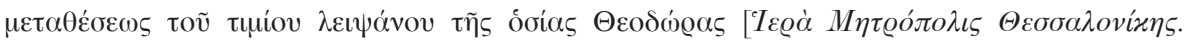

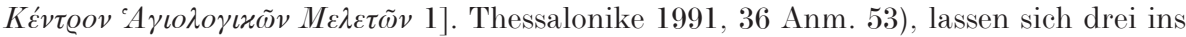
15. Jh. und eine ins 16. datieren; aus dem 15. Jh. stammen die Kodizes Mare. 506, Paris. 1213 und Vind. theol. 262 und aus dem 16. Jh. der Mosqu. 336 (Vladimir 213). Obwohl der Kodex Meteor. Barlaam 202 im Katalog ebenfalls ins 15. Jh. datiert wird, neige ich dazu, für diejenigen Teile, die Werke des Nikolaos Kabasilas tradieren, die von Laurent vorgeschlagenen Datierung ins 14. Jh. zu akzeptieren (vgl. die Diskussion über

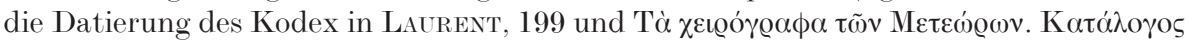

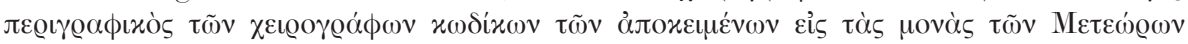

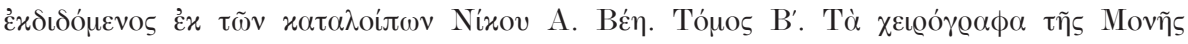

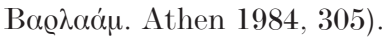

${ }^{14}$ Auf den ersten Blättern des Enkomions ist es nur dort möglich, die ältere Version des Textes wiederzugewinnen, wo der neue Text von Kabasilas, der an Stelle des ausradierten geschrieben ist, einen Teil der rasura freiläßt. Auf den späteren Folien zieht es Kabasilas vor, die zu ändernden Textpartien durch- oder querzustreichen und die neue Version in margine zu schreiben.

15 Die Zahlen verweisen auf die Ausgabe des Enkomions in der PG 150, 753-772, die als Vergleichsbasis benutzt wurde. Leider gibt die Ausgabe den Text des Kodex Vind. theol. 262 wieder, der seinerseits Apographon des Paris. $1213 \mathrm{zu}$ sein scheint, und fügt zu den falschen Lesarten des Kodex einige Druckfehler hinzu. 


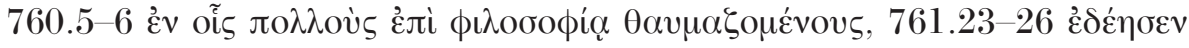

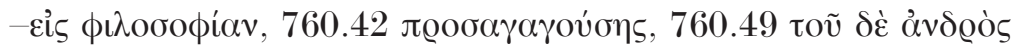

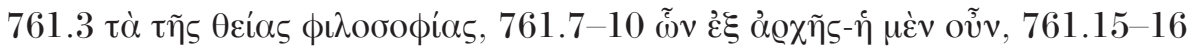

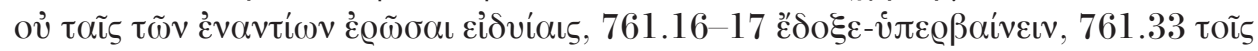

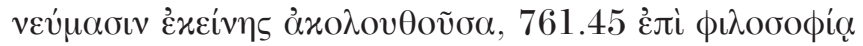

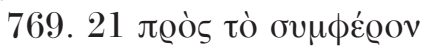

Der Umfang der Überarbeitung des Textes dokumentiert sich an mehreren Stellen, wo wir in der Lage sind, die ursprüngliche Version des Textes (Redaktion A) zu lesen. Einige Beispiele sind signifikant für die Art der Änderungen:

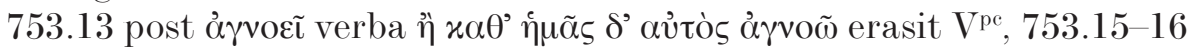

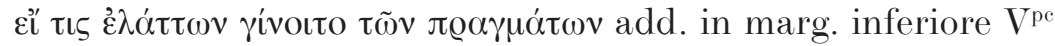

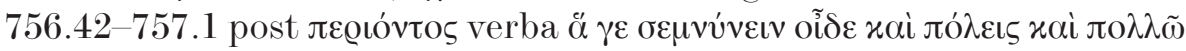

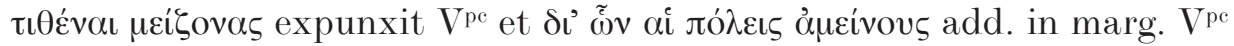

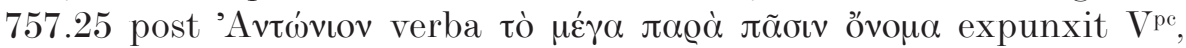

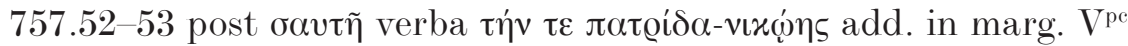

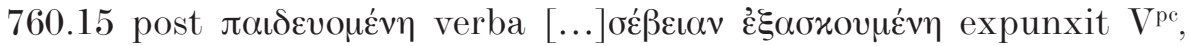
760.19 post

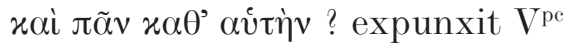

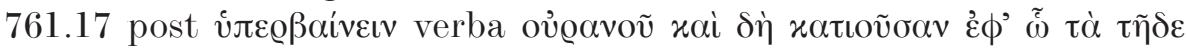

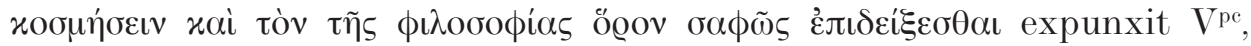

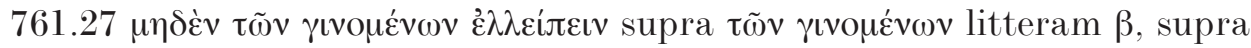

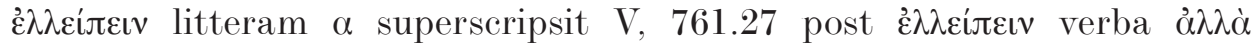

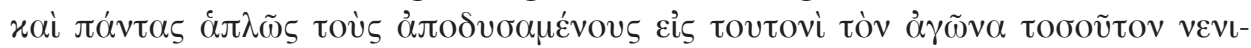

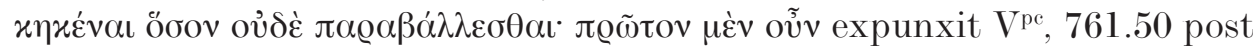

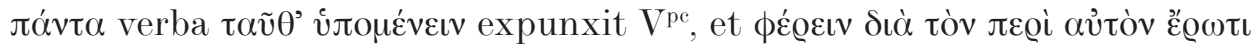

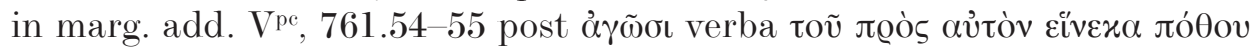
expunxit $V^{p c}$

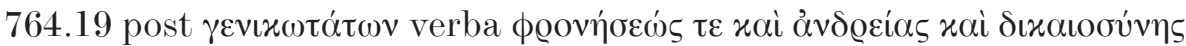

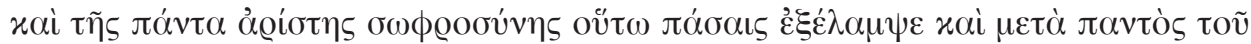

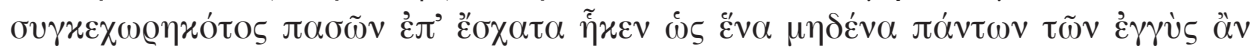

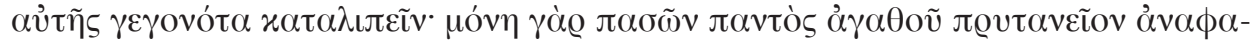

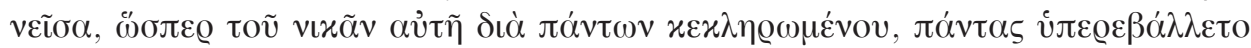
expunxit $V^{p e}$

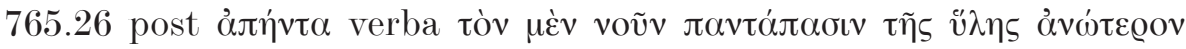

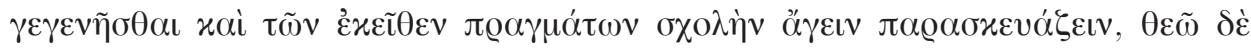

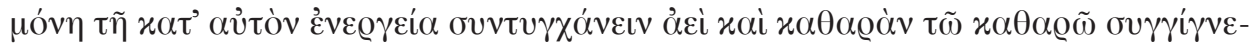

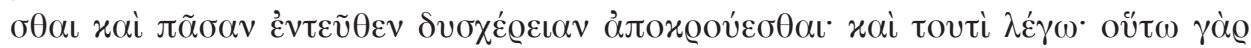

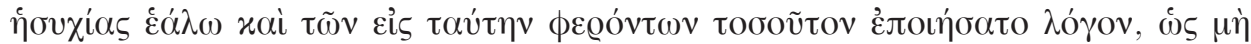

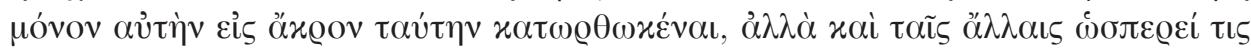

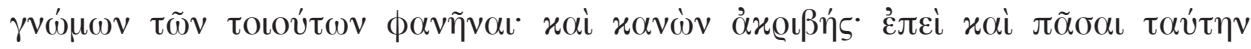




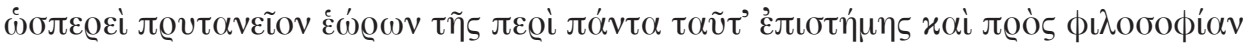

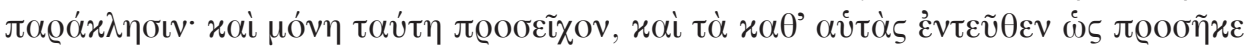

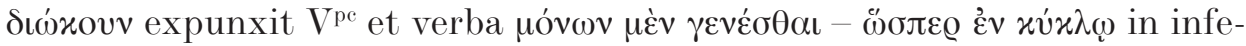
riore marg. fol. 93 add. $\mathrm{V}^{\mathrm{pc}}$ (cf. $\mathrm{PG} 765.26-32$ )

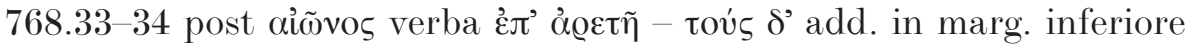

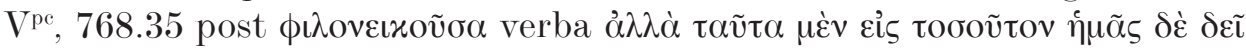

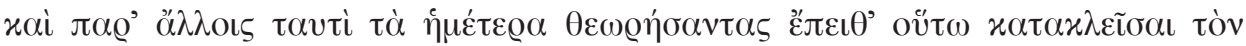
$\lambda$ ó $\gamma$ ov expunxit $V^{p c}$, et verba $\delta \tilde{\eta} \lambda o v ~ \delta \dot{\varepsilon}$ add. in marg. $V^{p c}$

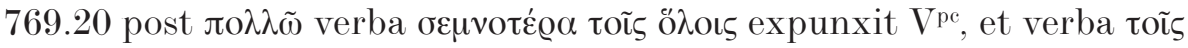

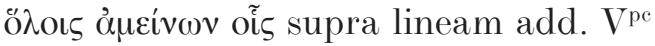

Von den post correctionem Änderungen des Palatinus hat der Schreiber des Kodex Meteor. Barlaam 202 (B) die meisten in den Text aufgenommen, einige dagegen nicht. Besonders beachtenswert sind die im letzten Teil des Enkomions befindlichen Änderungen des Palatinus, die der Kodex B nicht aufgenommen hat ${ }^{16}$. Nikolaos Kabasilas vergleicht in der ersten Fassung, wie viele seiner Vorgänger, die heilige Theodora mit früheren Heiligen und preist sie über alle Heilige. Bei dem Korrekturgang streicht er alle betreffenden Wörter bzw. Sätze durch und verringert damit sein Lob ${ }^{17}$ : 765.36-39 xai

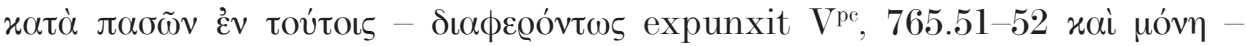

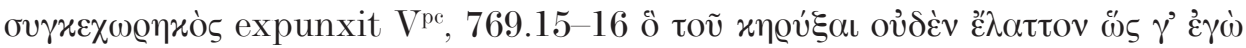

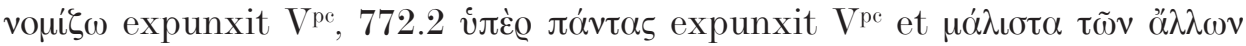

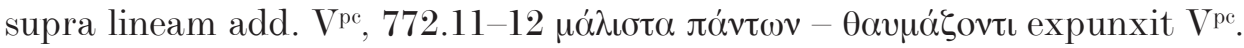

Eine Erklärung, warum Nikolaos Kabasilas sich für diese Herabsetzung seines Lobs entschieden hat, bietet uns möglicherweise ein Brief seines Onkels Neilos Kabasilas an ihn, in dem er die Reaktionen beschreibt, die das Enkomion des jungen Nikolaos Kabasilas auf den heiligen Demetrios bzw. der Vergleich des Märtyrers mit Johannes dem Täufer in Thessalonike

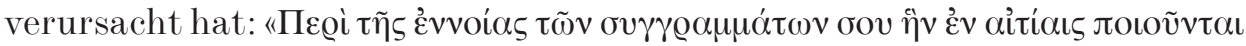

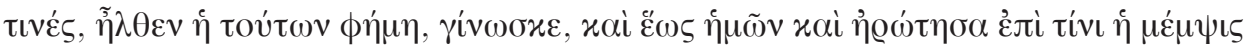

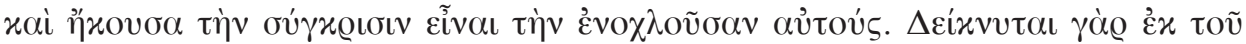

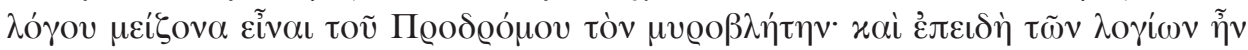

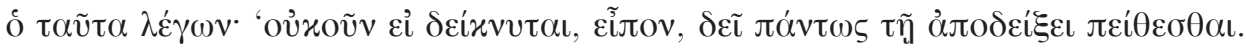

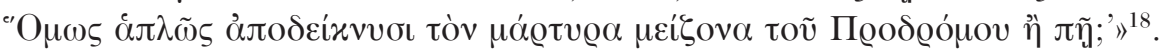

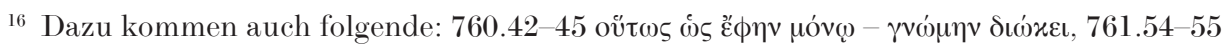

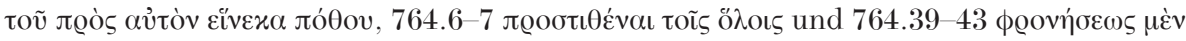

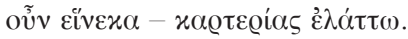

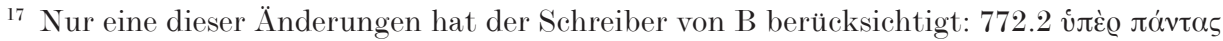

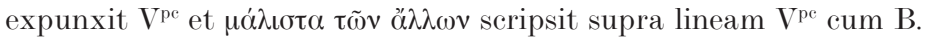

18 Den Brief, der dem Enkomion im Kodex Paris. 1213 folgt, hat Sp. Lambros ediert, s.

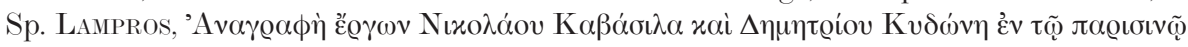

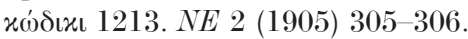


Wenn diese Beschuldigung Nikolaos Kabasilas zu den oben erwähnten Korrekturen veranlaßt hat, dann dürfen wir annehmen, daß die erste Fassung des Enkomion auf die heilige Theodora aus der gleichen Zeit wie das Enkomion auf den heiligen Demetrios herrührt.

Bezeichnend für das Verhältnis des Kodex Meteor. Barlaam 202 (B) ist, daß sein Schreiber einige der in $\mathrm{V}$ post correctionem getilgten Wörter bzw. Satzteile kopiert und nachträglich ebenfalls getilgt oder durchgestrichen hat. Dies erlaubt uns die Annahme, daß er beim Abschreiben möglicherweise den Kodex Palatinus vor sich hatte: 757.25 post 'Avtóvıov rasura in

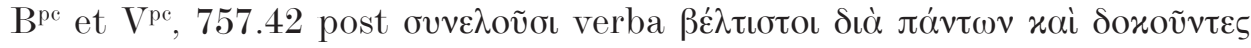

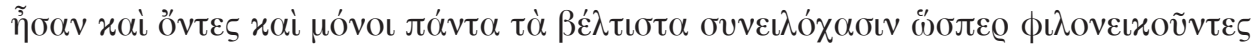

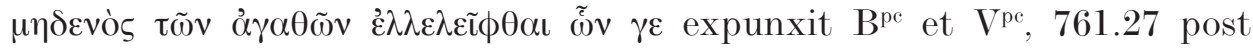

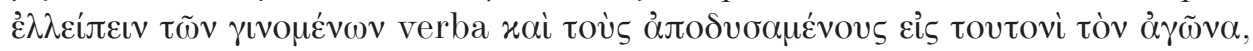

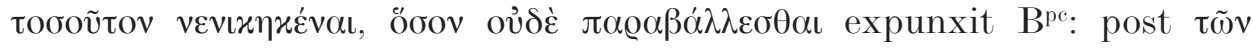

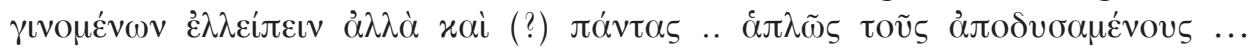
$\pi \alpha \varrho \alpha \beta \alpha \lambda \lambda \varepsilon \dot{\varepsilon} \sigma \theta \alpha$ expunxit $V^{p e}, 764.54$ post $\varepsilon \varepsilon \tau \eta \varrho \eta x v \tilde{\alpha} \alpha$ verba $\alpha u ̈ \tau \eta ~ \mu o ́ v \eta ~ \pi \alpha \sigma \tilde{\omega} v$

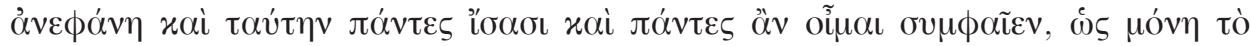

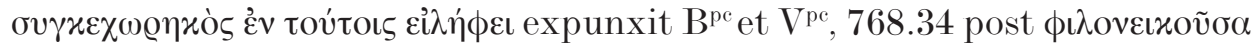

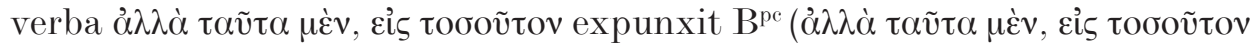

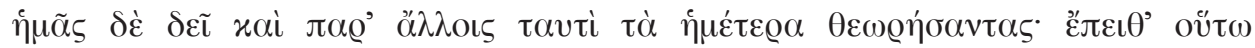

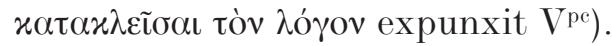

Darüber hinaus unterscheidet sich der Kodex B vom Palatinus durch einige Lesarten, die er gemeinsam mit den anderen Handschriften gegen $\mathrm{V}$ ante und post correctionem bietet; es handelt sich um kleine Änderungen, die als in scribendo Verbesserungen bzw. Verschlechterungen des Textes des Palatinus seitens des Kopisten von B gewertet werden können: 757.18 xai

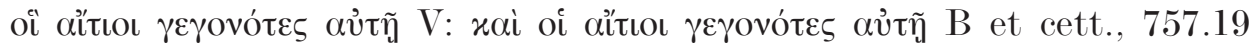

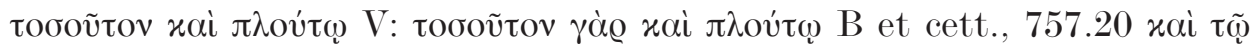

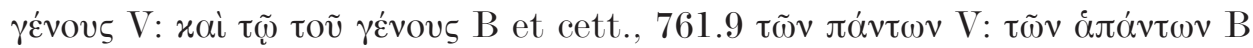

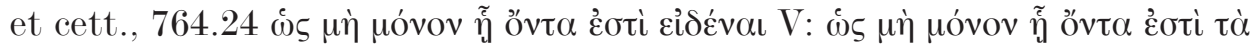

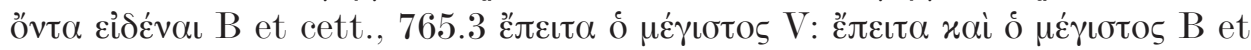

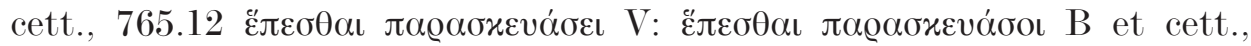

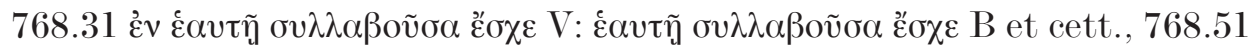

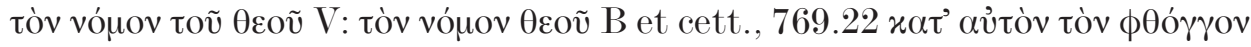

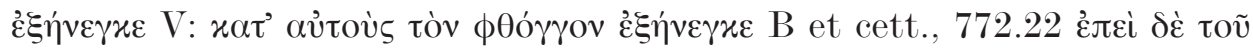

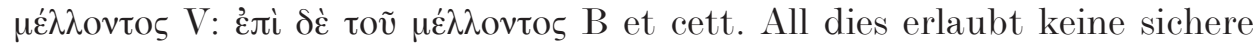
Aussage über die Identifizierung des Kopisten von B mit dem Verfasser des Enkomions, Nikolaos Kabasilas, schließt aber doch eine solche ${ }^{19}$ sowie auch

19 Diese kann nur anhand einer detailierten Untersuchung des Kodex B geschehen und liegt nicht im Rahmen dieses Aufsatzes. Es ist aber doch bewiesen, daß auch Auto- 
eine stemmatische Abhängigkeit des Kodex B von $V^{p e}$ (Redaktion B) nicht aus. B repräsentiert m.E. eine Reinschrift des von Nikolaos Kabasilas korrigierten Enkomions, in die einige Änderungen eingeflossen sind (Redaktion C), aus der alle weiteren Handschriften stammen ${ }^{20}$.

Die Identifizierung des Kopisten des Palatinus mit Nikolaos Kabasilas beantwortet darüber hinaus die Frage nach dem Paraphrasten der Vita der heiligen Theodora, die im Kodex von derselben Hand geschrieben ist. Ed. Kurtz hat in seiner Ausgabe mit Recht angenommen und bewiesen, «dass der Schreiber des Palatinus gleich während des Abschreibens die ... redaktionellen Änderungen am Text vorgenommen hat, dass also der Schreiber des Palatinus mit dem Urheber der in dieser Handschrift gebotenen abweichenden Rezension der anonymen Vita der hl. Theodora identisch ist $»^{21}$. Die Zuweisung dieser Paraphrase an Nikolaos Kabasilas, der selbst an der Vita der heiligen Theodora interessiert war, ist m.E. einwandfrei.

grapha Fehler haben können; vgl. dafür H. Hunger, Schreiben und Lesen im Byzanz. Die byzantinische Buchkultur. München 1989, 109-111.

${ }^{20}$ Ein ähnliches Schema s. auch bei ŠEvčEnko, 185.

${ }^{21}$ Ed. Kuntz, Des Klerikers Gregorios Bericht über Leben, Wunderthaten und Translation der hl. Theodora von Thessalonich nebst der Metaphrase des Joannes Staurakios [Mémoires de l'Académie impériale des sciences de St.-Pétersbourg, VIII ${ }^{e}$ sèrie, VI.1]. St. Pétersbourg 1902, VI. Die Vita ist auch von einem älteren Kodex, dem Mosqu. 159, überliefert (die Existenz einer dritten Handschrift aus dem Iberon-Kloster, Iberon 595 aus dem 16. Jh., ist für die Textkonstituierung irrelevant, da diese nur ein Apographon des Mosquensis ist; der Mosquensis befand sich bis zum 17. Jh. ebenfalls im IberonKloster). 